\title{
Do you remember proposing marriage to the Pepsi machine? False recollections from a campus walk
}

\author{
JOHN G. SEAMON, MORGAN M. PHILBIN, and LIZA G. HARRISON \\ Wesleyan University, Middletown, Connecticut
}

\begin{abstract}
During a campus walk, participants were given familiar or bizarre action statements (e.g., "Check the Pepsi machine for change" vs. "Propose marriage to the Pepsi machine") with instructions either to perform the actions or imagine performing the actions (Group 1) or to watch the experimenter perform the actions or imagine the experimenter performing the actions (Group 2). One day later, some actions were repeated, along with new actions, on a second walk. Two weeks later, the participants took a recognition test for actions presented during the first walk, and they specified whether a recognized action was imagined or performed. Imagining themselves or the experimenter performing familiar or bizarre actions just once led to false recollections of performance for both types of actions. This study extends previous research on imagination inflation by demonstrating that these false performance recollections can occur in a natural, real-life setting following just one imagining.
\end{abstract}

Are there limits on fictitious actions that we subsequently remember to be real? Considerable memory research, spurred initially by cases of recovered memories of abuse, has indicated that false memory for actions can be reliably demonstrated. For example, research has shown that when a plausible but false childhood event, such as getting lost in a mall, is embedded in a series of true childhood events, and those events are attributed to a trusted family source, adults can subsequently accept that false event as real (Loftus \& Pickrell, 1995). Still other studies have shown that more unusual fictitious childhood events, such as running around at a wedding and knocking a punchbowl onto the laps of the bride's parents, can also be falsely remembered by adults when the fictitious action is embedded in a set of real childhood events and attributed to the participants' parents (Hyman \& Pentland, 1996). Similarly, when childhood portraits are inserted into photographs to portray an event that never took place, such as a hot air balloon ride with a family member, adults can generate false recollections based on the altered photo (Wade, Garry, Read, \& Lindsay, 2002). Typically, such false memories occur when a fictitious event is presented in the context of real events from the distant past, the events are attributed to a trusted source, and, to aid in remembering, the participant is given time and instructions to imagine them repeatedly (for a review, see Brainerd \& Reyna, 2005).

Appreciation is expressed to Elizabeth Loftus, Henry Roediger, Maryanne Garry, David Balota, and an anonymous reviewer for helpful comments. Address correspondence to J. G. Seamon, Psychology Department, Wesleyan University, Middletown, CT 06459 (e-mail: jseamon@wesleyan.edu).
Other studies have bypassed the potential problem that a false childhood event might have actually occurred in the past by presenting participants with a series of actions to perform or imagine in a laboratory setting, and later testing the participants' recent memory for both the actions and the source of those actions. Goff and Roediger (1998), for example, presented participants with common objects as they heard simple action statements such as "break the toothpick," heard the statements and performed those actions, or heard the statements and imagined performing the actions. One day later, the participants were given some of the old objects and actions along with some new ones, and they were asked to imagine each action one, three, or five times. Two weeks later, the participants were given a list of actions taken from the first and second sessions, together with new actions, and asked to indicate which actions were presented in Session 1 and, if presented, whether those actions were imagined, performed, or only heard. These researchers found that increasing the number of imaginings of a new action in the second session increasingly led participants to misremember that action as having been performed in the first session, thereby demonstrating false recollection by imagination inflation (see also Sharman, Garry, \& Beuke, 2004). Subsequently, Thomas and Loftus (2002) and Thomas, Bulevich, and Loftus (2003) extended these false performance results with a similar procedure and showed that not only would participants misremember imagined common actions (e.g., flipping a coin), they would also falsely remember imagined bizarre actions (e.g., kissing a magnifying glass). Thomas et al. further demonstrated that false memories for performing familiar or bizarre actions could be enhanced when participants added perceptual and sensory details to their imagined actions. 
Together, these studies indicate that participants can sometimes misremember as having been performed actions that were merely imagined at an earlier time (see also Henkel, Franklin, \& Johnson, 2000; Mazzoni \& Memon, 2003).

The present research extended these imagination inflation studies by testing participants' memory for familiar or bizarre actions that were imagined or performed while the participants were walking in a familiar, natural environment. The use of a walk in a natural environment has a long history in the field of memory. For example, Galton (1879) used objects observed during his repeated walks in the Pall Mall section of London to trigger autobiographical recollections, and the ancient method of loci mnemonic has long been favored by memorists to learn associations by relational imagery between geographical locations and to-be-remembered information (Yates, 1966). In the present research, however, we sought to use geographical locations as a novel method of testing false memory by imagination inflation. We presented familiar and bizarre actions during long walks around a college campus to provide each action with a different context and more cues to help participants distinguish performed from imagined actions. To the extent that participants could use these cues, as memorists might use locations in the method of loci, the campus walk procedure might diminish the levels of false recollection observed previously in studies of imagination inflation. We examined false performance recollection by comparing participants who either performed or imagined performing familiar or bizarre actions themselves with participants who watched someone else or imagined someone else performing those actions.

\section{METHOD}

\section{Participants}

Forty Wesleyan University students either served as paid volunteers for $\$ 20$ each or received $3 \mathrm{~h}$ of psychology research credit.

\section{Materials}

For a walk, we selected 72 physical locations that included all areas of the Wesleyan campus. These locations were both indoors and outdoors: for example, a Pepsi machine in a lounge; the front stairs of the Psychology Building; a dictionary in the main library; and a couch in a reading room. In each location, one familiar action and one bizarre action would later be associated with that location (e.g., "Check the Pepsi machine for change"-"Get down on one knee and propose marriage to the machine"; "Wave from the top of the steps"-"Recite the balcony-scene lines from Romeo and Juliet"; "Look up a word in the dictionary"-"Pat the dictionary and ask it how it's doing"; "Lie down on the couch and relax"-"Lie down on the couch and talk to Freud"). No locations or actions were used more than once during a walk, and only one action was used for each location. Because one familiar action and one bizarre action came to be associated with each location, two sets of 72 locations and associated actions were constructed to counterbalance location with action type. Each set had 36 familiar and 36 bizarre actions randomly assigned to the locations.

\section{Procedure}

For an outline of our general procedure, see the Appendix. We told the participants that this three-session study concerned perception and imagination for typical and atypical actions that could occur during a campus walk. No mention was made of any memory test. In Session 1, the experimenter took the participant on a 1-h campus walk that included 48 locations, with 24 familiar and 24 bizarre actions randomly associated with these locations. The experimenter read one action statement at each stop, and the participants either performed that action, watched the experimenter perform it, vividly imagined themselves performing it for $10 \mathrm{sec}$, or vividly imagined the experimenter performing it for $10 \mathrm{sec}$. One group of 20 participants performed half of the actions and imagined performing the remaining actions themselves, whereas another group of 20 participants watched an experimenter perform half of the actions and imagined the experimenter performing the remaining half.

In Session 2, 1 day later, the experimenter and participant took a second campus walk involving 36 locations along the same route. This walk included 12 locations from Session 1 (where actions were previously performed), 12 additional locations from Session 1 (where actions were previously imagined), and 12 new locations along the route with actions that were not previously used. Repeated actions were always the same. Each subset of 12 locations had six familiar and six bizarre associated actions. All actions were now imagined for $10 \mathrm{sec}$ and rated for vividness on a 5-point scale. The participants who had previously performed or imagined actions in Session 1 now imagined themselves performing each action, whereas the participants who had previously observed or imagined the experimenter performing actions now imagined the experimenter performing all actions. Following Thomas et al. (2003), the experimenter asked two perceptual questions after each action statement was read (e.g., when asked to imagine knocking on a campus door, the participant was also asked "What sound does your knocking make?" and "What do your knuckles feel like as you knock?") to strengthen the perceptual-sensory details of the imagined actions.

In Session 3, conducted 2 weeks later in the memory lab, the participants were given two memory tests for their first campus walk. The first test consisted of 72 action statements in which the participants were instructed to indicate whether or not each action was presented in Session 1 and, if so, whether it was imagined or performed. In addition, the participants were instructed to indicate on a 5-point scale their confidence in both decisions. The 72 actions, half of them familiar and half of them bizarre, were obtained from the following conditions and presented in random order: (1) 12 actions performed in Session 1 and imagined in Session 2 (performed-imagined); (2) 12 actions performed in Session 1, but not used in Session 2 (performed-not used); (3) 12 actions imagined in Sessions 1 and 2 (imagined-imagined); (4) 12 actions imagined in Session 1, but not used in Session 2 (imagined-not used); (5) 12 actions not used in Session 1, but imagined in Session 2 (not used-imagined); and (6) 12 filler actions not used in either session (not used-not used). Accurate recognition memory for actions presented in Session 1 is indicated by positive responses to actions from the performed-imagined, performed-not used, imagined-imagined, and imagined-not used conditions; accurate performance memory for those actions is indicated by performance responses only from the performed-imagined and performed-not used conditions. False recognition memory, in which actions were wrongly remembered as presented in Session 1, is indicated by positive responses to actions from the not used-imagined and not used-not used conditions; whereas false performance memory, in which actions were wrongly remembered as performed in Session 1, is indicated by performance responses to actions from the imagined-imagined, imagined-not used, not used-imagined, and not used-not used conditions.

The second memory test involved a location-action matching task to gauge the degree to which the participants learned associations between locations and actions from the walks. The participants were presented with a list of the 48 locations used in Session 1 in their order of appearance during the walk, and a second list containing the 48 actions used in Session 1, arranged in a random order and numbered from 1 to 48 . The participants were instructed to match each action to its correct location by writing the action number next to the appropriate location.

\section{RESULTS}

Table 1 presents the action-recognition findings ("Was this action presented?") from Session 1. The participants 
demonstrated strong recognition and high confidence for both familiar and bizarre actions from Session 1 if those actions were presented during both campus walks (the performed-imagined and imagined-imagined conditions). For actions presented only during the first walk (the performed-not used and imagined-not used conditions), lower recognition and confidence were shown than for actions presented twice, and there was little difference in recognition for actions imagined once during either the first or second walk (the imagined-not used and not used-imagined conditions). Actions not used in either walk yielded minimal recognition (the not used-not used condition). These results were supported by ANOVAs, and all differences were statistically significant at least at the .05 confidence level. Because memory for actions and source judgments was unaffected by whether the participants performed or imagined performing the actions themselves (Group 1) or watched or imagined the experimenter performing the actions (Group 2), all results from the two experimental groups were pooled. (The between-participants variable experimental group was included in all analyses, with a significant but nonsystematic difference involving this variable observed in only 6 of 52 tests.) Moreover, these data were not corrected for guessing, because the false alarm rates for familiar and bizarre actions were equivalent and low. Action recognition was greater for the performedimagined condition than for the performed-not used condition $\left[F(1,38)=25.38, M S_{\mathrm{e}}=0.81\right]$, and bizarre actions were recognized more than familiar actions $[F(1,38)=$ $\left.10.80, M S_{\mathrm{e}}=0.21\right]$. Action recognition was also higher in the imagined-imagined condition than in the imaginednot used condition $\left[F(1,38)=158.74, M S_{\mathrm{e}}=4.25\right]$, and bizarre actions were again recognized more than familiar actions $\left[F(1,38)=29.32, M S_{\mathrm{e}}=0.67\right]$. Finally, false action recognition was higher in the not used-imagined condition than in the not used-not used condition $[F(1,38)=$ 121.31, $\left.M S_{\mathrm{e}}=9.73\right]$, with no effect of action type. The high levels of false recognition demonstrated in the not used-imagined condition for familiar (.50) and bizarre (.57) actions indicate that participants frequently remembered imagined actions presented only in the second walk as actions presented in the first walk. Table 2 provides additional insight by showing how our participants actually remembered these falsely recognized actions.

Table 2 presents the results for the source monitoring judgments for recognized actions ("Was this action imagined or performed?") from Session 1. These judgments represent the primary focus of this study, and they demonstrate that our participants confused performance with imagination. Table 2 shows that recognized actions were judged more frequently as performed than imagined in the performedimagined and performed-not used conditions, whereas they were judged more often as imagined than performed in the imagined-imagined, imagined-not used, and not usedimagined conditions. The performed-imagined condition yielded more performed judgments than the performed-not used condition $\left[F(1,38)=14.27, M S_{\mathrm{e}}=0.62\right]$, with more performed judgments for bizarre than for familiar actions $\left[F(1,38)=35.87, M S_{\mathrm{e}}=0.76\right]$. The imagined-imagined
Table 1

Mean Proportions and Confidence Ratings (CRs) of Actions Recognized From Session 1 As a Function of Action Type and Action Presentation Condition

\begin{tabular}{|c|c|c|c|c|}
\hline \multirow{3}{*}{$\begin{array}{l}\text { Action Presentation } \\
\text { (Session 1-Session 2) }\end{array}$} & \multicolumn{4}{|c|}{ Action Type } \\
\hline & \multicolumn{2}{|c|}{ Familiar } & \multicolumn{2}{|c|}{ Bizarre } \\
\hline & $M$ & $\overline{\mathrm{CR}}$ & $M$ & $\overline{\mathrm{CR}}$ \\
\hline Performed-imagined & .92 & 4.64 & .95 & 4.84 \\
\hline Performed-not used & .74 & 4.46 & .85 & 4.19 \\
\hline Imagined-imagined & .90 & 4.46 & .94 & 4.60 \\
\hline Imagined-not used & .48 & 3.85 & .70 & 3.99 \\
\hline Not used-imagined & .50 & 4.04 & .57 & 4.03 \\
\hline Not used-not used & .04 & 2.54 & .05 & 3.35 \\
\hline
\end{tabular}

Note-CRs were based on a 5-point scale, where 1 indicated low and 5 high confidence.

condition yielded more imagined judgments than the imagined-not used condition $\left[F(1,38)=101.49, M S_{\mathrm{e}}=\right.$ 3.37], with more imagined judgments for bizarre than for familiar actions $\left[F(1,38)=21.38, M S_{\mathrm{e}}=0.73\right]$. The comparisons of the imagined-imagined, imagined-not used, and not used-imagined conditions with the not used-not used condition provide the critical data on imagination inflation. The imagined-imagined condition yielded more imagined $\left[F(1,38)=599.39, M S_{\mathrm{e}}=25.37\right]$ and performed $\left[F(1,38)=13.36, M S_{\mathrm{e}}=0.24\right]$ judgments than did the not used-not used condition. Similarly, the imagined-not used condition also yielded more imagined $[F(1,38)=360.40$, $\left.M S_{\mathrm{e}}=10.24\right]$ and performed $\left[F(1,38)=9.84, M S_{\mathrm{e}}=\right.$ $0.07]$ judgments than did the not used-not used condition. For the imagined-imagined and imagined-not used conditions, imagined judgments were more frequent for bizarre than for familiar actions (both $p \mathrm{~s}<.05$ ).

Of particular interest is the comparison between the not used-imagined and not used-not used conditions. The actions in the not used-imagined condition were merely imagined once in the second session. If participants believed that they actually performed these actions in the first session, this finding would provide a strong demonstration of false memory by imagination inflation under real-life conditions. Our results showed more imagined $\left[F(1,38)=82.29, M S_{\mathrm{e}}=6.38\right]$ and performed $[F(1,38)=$ $\left.13.20, M S_{\mathrm{e}}=0.27\right]$ judgments in the not used-imagined

Table 2

Mean Proportions of Imagined and Performed Actions Recognized From Session 1, and Confidence Ratings (CRs), As a Function of Action Type and Action Presentation Condition Action Type and Source Identification

\begin{tabular}{|c|c|c|c|c|c|c|c|c|}
\hline \multirow{3}{*}{$\begin{array}{l}\text { Action Presentation } \\
\text { (Session 1-Session 2) }\end{array}$} & \multicolumn{8}{|c|}{ Action Type and Source Identification } \\
\hline & \multicolumn{2}{|c|}{$\begin{array}{l}\text { Imagined } \\
\text { Familiar }\end{array}$} & \multicolumn{2}{|c|}{$\begin{array}{l}\text { Performed } \\
\text { Familiar }\end{array}$} & \multicolumn{2}{|c|}{$\begin{array}{c}\text { Imagined } \\
\text { Bizarre }\end{array}$} & \multicolumn{2}{|c|}{$\begin{array}{c}\text { Performed } \\
\text { Bizarre }\end{array}$} \\
\hline & $M$ & $\mathrm{CR}$ & $M$ & CR & $M$ & $\mathrm{CR}$ & $M$ & CR \\
\hline Performed-imagined & .15 & 3.82 & .77 & 4.52 & .05 & 3.84 & .91 & 4.78 \\
\hline Performed-not used & .09 & 3.42 & .64 & 4.60 & .07 & 3.34 & .78 & 4.48 \\
\hline Imagined-imagined & .78 & 4.36 & .12 & 4.32 & .84 & 4.36 & .10 & 4.05 \\
\hline Imagined-not used & .42 & 3.93 & .07 & 3.36 & .63 & 4.11 & .08 & 3.66 \\
\hline Not & .38 & 3.92 & .10 & 3.91 & .45 & 3.93 & .12 & 3.08 \\
\hline Not used-not used & .01 & 3.00 & .03 & 2.46 & .03 & 4.21 & .02 & 2.88 \\
\hline
\end{tabular}

Note-CRs were based on a 5-point scale, where 1 indicated low and 5 high confidence. 
condition than in the not used-not used condition. The level of false performance judgments of .10 to .12 for both familiar and bizarre actions after a single imagining, with moderately high confidence ratings, is similar in magnitude to that typically observed in laboratory studies of imagination inflation (e.g., Goff \& Roediger, 1998; Thomas et al., 2003; Thomas \& Loftus, 2002). ${ }^{1}$

Finally, the results of the location-action matching task demonstrated strong memory for locations and their associated actions from Session 1, with an average of .88 correct matches over all participants. This result from our delayed matching test is impressive and mimics the high level of performance typically observed over short or long retention intervals in studies in which participants are specifically instructed to employ the method of loci procedure (e.g., De Beni \& Cornoldi, 1985; Ross \& Lawrence, 1968).

\section{DISCUSSION}

We found that imagining familiar or bizarre actions during a campus walk can lead to the subsequent false recollection of having performed those actions. This finding is consistent with previous research involving imagination inflation for actions performed or imagined in a memory lab (e.g., Goff \& Roediger, 1998; Mazzoni \& Memon, 2003; Thomas \& Loftus, 2002), and it extends that research by demonstrating that comparable levels of false recollection can be obtained in a natural, changing environment. Apparently, one vivid imagining during a walk that focuses on the perceptual or sensory details of the image is sufficient to sometimes establish a false recollection for a familiar or bizarre action 2 weeks later. Also, studies of enactment typically show stronger memory accuracy for self-performed tasks than for experimenter-performed tasks (e.g., Mulligan \& Hornstein, 2003). However, unlike the present study, previous enactment studies varied self-performed and experimenter-performed tasks as a within-participants variable, not as a between-participants variable. This procedural difference, along with possible ceiling effects in the present performed conditions, may have contributed to our lack of enactment effect. For false recollection, imagining someone else performing an action was just as effective as imagining performing it yourself, ${ }^{2}$ and, consistent with prior research (Thomas \& Loftus, 2002), bizarre actions that were performed or imagined were recognized better than familiar actions, since their bizarreness enhanced their memorability. However, unlike that previous research, we did not find greater false performance recollection for familiar than for bizarre actions, indicating that caution is needed with this finding until more observations are available. Finally, these falserecollection results cannot be attributed to poor learning, because on a matching test our participants demonstrated strong learning for most location-action associations.

These results have important implications for falsememory research. We demonstrated that the phenomenon of false performance recollection by imagination inflation is observable in a natural, real-life setting, after a single imaging. Vivid imagining during a campus walk was suffi- cient sometimes to lead participants to claim that they had actually performed different familiar or bizarre acts that they had merely imagined doing previously, such as waving from a balcony or proposing marriage to a Pepsi machine. These results are interpretable in terms of a sourcemonitoring framework in which participants confuse vivid perceptual elements of a recalled image with a memory for an action (Johnson, Hashtroudi, \& Lindsay, 1993).

We believe that our results also add to the growing literature that warns of the danger of false recollection when imagination is used as a memory aid. One might initially diminish the importance of imagination inflation in this context because of the modest size of this effect. For example, studies typically find levels of false performance recollection (.10 to .20) that are far below the levels of accurate performance recollection (e.g., Goff \& Roediger, 1998; Thomas et al., 2003; Thomas \& Loftus, 2002; the present research). In this regard, false memory by imagination inflation is quite different from some other memory illusions, such as the Deese, Roediger, and McDermott illusion involving converging semantic associates, that can produce comparable levels of accurate and false memory (e.g., Roediger \& McDermott, 1995; Seamon et al., 2006). Given that different illusions can produce effects of varying magnitude, the relative size of imagination inflation should not diminish its importance, because this effect can have serious implications for autobiographical recollection. To the extent that studies of guided imagery and imagination inflation demonstrate that false recollections of performance can follow imagination, these studies display the need for caution in the use of imagery to recover memories. Of course, the use of imagery to aid memory retrieval is different from the use of imagery for encoding actions, and this observation might suggest little common ground for imagination as an aid to memory recovery and studies of imagination inflation that use imagery to encode actions. However, if imagery for an action or event is used over an extended period of time, the clear distinction between encoding and retrieval can be blurred. It may be impossible to know the level of false recollection generated by imagery in everyday life, but it is clear from empirical research on memory in general, and studies of guided imagery and imagination inflation in particular, that false alarm rates are almost never zero. False performance recollection for both familiar and bizarre actions is real. The present research extends previous work by demonstrating that these false recollections can sometimes occur in a natural, real-life setting following just one imagining.

\section{REFERENCES}

Brainerd, C. J., \& Reyna, V. F. (2005). The science of false memory. New York: Oxford University Press.

DE BENI, R., \& CoRNOLDI, C. (1985). Effects of the mnemotechnique of loci in the memorization of concrete words. Acta Psychologica, 60, 11-24.

Galton, F. (1879). Psychometric experiments. Brain, 2, 149-162.

Goff, L. M., \& Roediger, H. L., III (1998). Imagination inflation for action events: Repeated imaginings lead to illusory recollections. Memory \& Cognition, 26, 20-33.

Henkel, L. A., Franklin, N., \& Johnson, M. K. (2000). Cross-modal source monitoring confusions between perceived and imagined 
events. Journal of Experimental Psychology: Learning, Memory, \& Cognition, 26, 321-335.

Hyman, I. E., JR., \& Pentland, J. (1996). The role of mental imagery in the creation of false childhood memories. Journal of Memory \& Language, 35, 101-117.

Johnson, M. K., Hashtroudi, S., \& Lindsay, D. S. (1993). Source monitoring. Psychological Bulletin, 114, 3-28.

Loftus, E. F., \& Pickrell, J. E. (1995). The formation of false memories. Psychiatric Annals, 25, 720-725.

Mazzoni, G., \& Memon, A. (2003). Imagination can create false autobiographical memories. Psychological Science, 14, 186-188.

Mulligan, N. W., \& Hornstein, S. L. (2003). Memory for actions: Self-performed tasks and the reenactment effect. Memory \& Cognition, 31, 412-421.

Roediger, H. L., III, \& McDermott, K. B. (1995). Creating false memories: Remembering words not presented in lists. Journal of Experimental Psychology: Learning, Memory, \& Cognition, 21, 803-814.

Ross, J., \& LaWrence, K. A. (1968). Some observations on memory artifice. Psychonomic Science, 13, 107-108.

Seamon, J. G., Berko, J. R., Sahlin, B., Yu, Y.-L., Colker, J. M., $\&$ GotTFRIED, D. H. (2006). Can false memories spontaneously recover? Memory, 14, 415-423.

Sharman, S. J., Garry, M., \& Beuke, C. J. (2004). Imagination or exposure causes imagination inflation. American Journal of Psychology, 117, 157-168.

Thomas, A. K., Bulevich, J. B., \& Loftus, E. F. (2003). Exploring the role of repetition and sensory elaboration in the imagination inflation effect. Memory \& Cognition, 31, 630-640.

Thomas, A. K., \& LofTus, E. F. (2002). Creating bizarre false memories through imagination. Memory \& Cognition, 30, 423-431.

Wade, K. A., Garry, M., Read, J. D., \& Lindsay, D. S. (2002). A picture is worth a thousand lies: Using false photographs to create false childhood memories. Psychonomic Bulletin \& Review, 9, 597-603.

Yates, F. A. (1966). The art of memory. Chicago: University of Chicago Press.

\section{NOTES}

1. In our study, two imaginings of actions in separate sessions did not yield more false performance memory than did a single imagining, whereas previous research found that multiple imaginings in a single session produced more performance errors than did a single imagining (Goff \& Roediger, 1998; Thomas \& Loftus, 2002). Although multiple spaced imaginings were not feasible in our study (a walk took $1 \mathrm{~h}$ ), it is conceivable that three to five imaginings over multiple walks could enhance performance errors, as was found in the laboratory studies.

2. The only conditions that permitted testing enactment were the performed-imagined and performed-not used conditions. We tested whether participant-performed actions were recognized better than experimenter-performed actions and found that recognition was greater for performed-imagined actions than for performed-not used actions $\left[F(1,38)=25.38, M S_{\mathrm{e}}=0.81\right]$, but that there was no effect of enactment for both action conditions together or for either condition alone (all $F_{\mathrm{s}}<1$ ). Enactment in this study was only varied in a between-participants context.

\section{ARCHIVED MATERIALS}

The following materials associated with this article may be accessed through the Psychonomic Society's Norms, Stimuli, and Data archive, www.psychonomic.org/archive/.

To access these files, search the archive for this article using the journal name (Psychonomic Bulletin \& Review), the first author's name (Seamon), and the publication year (2006).

\section{FILE: Seamon_PB\&R-2006.zip}

DESCRIPTION: The compressed archive file contains two files:

Session1VersionA.doc, containing the locations and actions from the Wesleyan University campus used by Seamon et al. in Session 1.

Session2VersionA.doc, containing the locations and actions from the Wesleyan University campus used by Seamon et al. in Session 2 .

AUTHOR's E-MAIL ADDRESS: jseamon@wesleyan.edu.

\section{APPENDIX \\ Outline of the Three Experimental Sessions}

\section{Day 1: First Walk}

Procedure. On a campus walk, stop at 48 locations and listen to 48 action statements ( 24 familiar, 24 bizarre).

Within-participants variables. Action type (familiar or bizarre); action status (performed or imagined).

Between-participants variable. Participant groups (Group 1 or 2).

Group 1: Participant actor. Perform 24 actions; imagine yourself performing 24 actions (half familiar, half bizarre).

Group 2: Experimenter actor. Watch Experimenter perform 24 actions; imagine Experimenter performing 24 actions (half familiar, half bizarre).

\section{Day 2: Second Walk}

Procedure. Take another walk along the same route and listen to 36 action statements, 24 locations and actions from the first walk, and 12 new locations and actions (half familiar, half bizarre).

Within-participants variables. Action type (familiar or bizarre); action status (previously performed, previously imagined, or new).

Between-participants variable. Participant groups (Group 1 or 2).

Group 1: Participant actor. Imagine yourself performing 12 previously performed, 12 previously imagined, and 12 new actions (half familiar, half bizarre).

Group 2: Experimenter actor. Imagine Experimenter performing 12 previously performed, 12 previously imagined, and 12 new actions (half familiar, half bizarre).

\section{Day 15: Memory Tests}

Procedure. All participants take the same memory tests. First, recognition and source monitoring tests of 72 actions, including 48 actions from Day 1, 12 additional actions from Day 2, and 12 new actions.

- Was it presented on the first walk?

- If yes, was it imagined or performed?

Second, location-action matching test of 48 locations and 48 actions from Day 1. Match actions to correct locations. 